\title{
From the Desk of Editor-in-Chief
}

Greetings to the readers and supporters of the Hypertension Journal, and our best wishes for 2017. I am pleased to report to the editorial board and the readers that the journal had a remarkable 2016 in terms of visibility, appeal, respect, and national recognition. I want to thank the publishers, the editorial board, and reviewers for the success of the journal. And we will make additional progress, be assured.

This issue of the journal has an excellent assembly of articles, views, and themes from physiology to diagnosis, to management, and to clinical applications, connected to hypertension for the benefit of the readers. Of special note is the scientific re-discovery of an old drug "chlorthalidone"; the latest scientific basis for the renewed

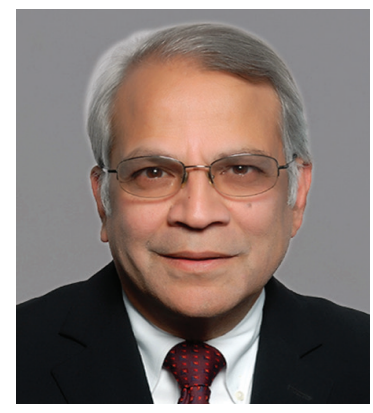
interest in this molecule is covered by world renowned experts in the field. Besides drug therapy, this issue also covers hypotheses and device-based therapy for hypertension. Thus, we are fulfilling the readers' expectations and needs for improved management of hypertension in the community.

With best wishes

C Venkata S Ram MD MACP FACC FASH

Editor-in-Chief

Hypertension Journal

Director, Apollo Institute for Blood Pressure Management Director, Blood Pressure Clinics, Apollo Group of Hospitals Andhra Pradesh, Hyderabad, India

Director, Texas Blood Pressure Institute, Dallas, Texas, USA

Director for South Asia Region

World Hypertension League (in partnership with WHO) 